Demgegenüber ist die NeugeborenenMorbidität (z.B. respiratorische Anpassungsstörungen, Apnoe, Bradykardie, Hypoglykämien, neurologische Defizite) in der 40. Schwangerschaftswoche mit $2,5 \%$ am niedrigsten; in der 37 . Woche ist das Morbiditätsrisiko mit 5,9\% noch mehr als verdoppelt [Shapiro-Mendoza CK et al. Pediatrics 2008; 121: e223-32]. Nach den Daten mehrerer Untersuchungen ist die Wahrscheinlichkeit für Tod oder schwere perinatale Komplikationen in der 37. Schwangerschaftswoche gegenüber der 39. Woche um über das Doppelte erhöht. Ramsauer wies darauf hin, dass bundesweit in $20 \%$ der Fälle die Dauer der Einlingsschwangerschaften 37 bis 38 Wochen beträgt [Poets CF et al. Dtsch Ärztebl Int 2012; 109: 721-6].

Wichtig ist bei der Wahl des Geburtstermins auch das "klinische Setting" mit der Verfügbarkeit geburtshilflicher, neonatologischer oder im Bedarfsfall auch kinderchirurgischer Abteilungen. Nicht zuletzt spielen Wohnort, ein möglicher Erschöpfungszustand sowie persönliche Wünsche der Schwangeren eine wichtige Rolle.

Dr. Andreas Fischer

3. Hauptthema Geburtshilfe, Ramsauer B:

"Geplanter Kaiserschnitt - optimaler Zeitpunkt", Donnerstag, 7. März 2013

\title{
Netze in der Beckenboden-Chirurgie schon Standard?
}

— Seit 1995 sind weltweit in der Inkontinenz-Behandlung mehr als 3 Mio. TensionFree-Vaginal-Tapes (TVT) eingesetzt worden. Prof. Heinz Kölbl, Universitätsklinikum Wien, verdeutlichte, dass damit knapp 80\% der Patientinnen wieder kontinent wurden und bei weiteren 20\% eine Besserung eintrat.

Einige Gründe sprechen dafür, alloplastische Implantate in Form von Netzen auch bei Beckenboden-Prolaps anzuwenden. Kölbl nannte neben den guten Erfahrungen mit der TVT-Technik die Beobachtung, dass die autologe Rekonstruktion oft ohne dauerhaften Erfolg ist und Rezidivraten bis zu $50 \%$ zeigt. Häufig kann für eine autologe
Rekonstruktion aus dem Spatium rectovaginale teilweise auch gar kein Material entnommen werden.

Problematisch ist allerdings die höhere Komplikationsrate. So zeigte eine Studie, dass der transvaginale Einsatz von Netzen gegenüber einer anterioren Kolporrhapie zwar mit kürzerer Operationszeit und geringerem Blutverlust durchgeführt werden kann - Komplikationen und erneute Eingriffe waren jedoch deutlich höher: 3,5 vs. $0,5 \%$ Blasenperforation, 12,3 vs. $6,3 \%$ erneute Stressinkontinenz, 3,0 vs. $0 \%$ ReOperation wegen Mesh-Exposition [Altmann D et al. N Engl J Med 2011; 364: 1826-36]. Die US-amerikanische FDA hat

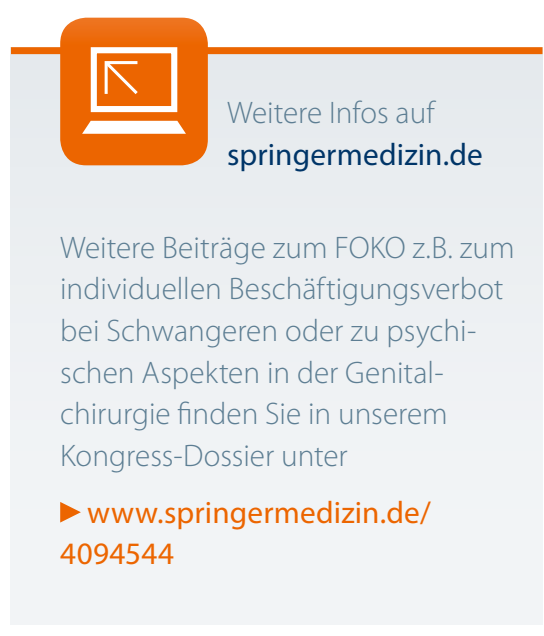

2011 erneut vor dem Einsatz von Netzen bei Beckenboden-Prolaps gewarnt und auf Netzerosionen mit Häufigkeiten bis zu $35,1 \%$, Schmerzen $(31,4 \%)$, Infektionen $(16,8 \%)$ oder Dyspareunie $(7,2 \%)$ hingewiesen. Alloplastische Materialien bezeichnete Kölbl als wichtigen Bestandteil des operativen Repertoires, vor allem bei Rezidiven. Neue Produkte sollten jedoch nur innerhalb von kontrollierten Studien eingesetzt werden. Die Risiken müssten ausführlich mit den Patientinnen besprochen werden.

Dr. Andreas Fischer

5. Hauptthema Gynäkologie/Urogynäkologie, Kölbl H: „Aktueller Stand über den Einsatz von Netzen bei urogynäkologischen Operationen", Freitag, 8. März 2013

Familienplanung und hat ein solitäres symptomatisches Myom. An Nebenwirkungen sind transiente Amenorrhö (bis 10\%), permanente Amenorrhö (bis $14 \%$ bei Patientinnen über 45 Jahren) sowie ein Postembolisationssyndrom bekannt. Bislang nur wenige Zentren bieten die MRT-gesteuerte Zerstörung des Myoms mit hochfrequentem Ultraschall (HIFU) an. Die Prozedur dauert zwischen zwei und drei Stunden. An Komplikationen nannte Becker vor allem Entzündungsreaktionen des subkutanen Fettgewebes oder der Bauchmuskulatur (bis $11 \%$ ) sowie Hautverbrennungen (bis $5 \%$ ).

Dr. Andreas Fischer

5. Hauptthema Gynäkologie/Urogynäkologie, Becker S: „Interdisziplinäre Myomtherapie worum geht es eigentlich“, Freitag, 8. März 2013 